\title{
Change in biomarkers of type- 2 inflammation following severe exacerbations of asthma
}

\author{
Ruth Semprini, ${ }^{1,2}$ Nick Shortt, ${ }^{1,2}$ Stefan Ebmeier, ${ }^{1}$ Alex Semprini, ${ }^{1,2}$ Rachel Varughese, ${ }^{1}$ \\ Cecile T J Holweg, ${ }^{3}$ John G Matthews, ${ }^{3}$ James Fingleton, 1,4 Mark Weatherall, 1,4,5 \\ Richard Beasley, 1,2,4 Irene Braithwaite ${ }^{1,4}$
}

\begin{abstract}
- Additional material is published online only. To view please visit the journal online (http://dx.doi.org/10.1136/ thoraxjnl-2018-211657).

${ }^{1}$ Medical Research Institute of New Zealand, Wellington, New Zealand

${ }^{2}$ Victoria University Wellington, Wellington, New Zealand

${ }^{3}$ Genentech Inc, South San Francisco, California, USA

${ }^{4}$ Capital \& Coast District Health Board, Wellington, New Zealand ${ }^{5}$ University of Otago, Wellington, New Zealand
\end{abstract}

\section{Correspondence to}

Dr Irene Braithwaite, Medical

Research Institute of New

Zealand, Wellington 6242, New Zealand;

irene.braithwaite@mrinz.ac.nz

Received 7 February 2018

Revised 28 May 2018

Accepted 2 July 2018

Published Online First

18 July 2018

\begin{abstract}
We investigated the time course of change of type-2 asthma biomarkers after a severe asthma exacerbation. Blood eosinophils were lowest immediately after treatment was initiated $\left(0.07\right.$ vs $\left.0.33 \times 10^{9} / \mathrm{L}, \mathrm{p}<0.001\right)$ while serum IgE levels were at their highest (339 vs 249 $U / L, p<0.001)$. Fractional exhaled Nitric Oxide levels were lowest 2 weeks after treatment ( 23 vs 33 ppb, $p=0.06)$ and serum periostin levels were lowest 1 week after treatment $(45.9$ vs $50.9 \mathrm{ng} / \mathrm{mL}, \mathrm{p}<0.001)$. A delay of $4-8$ weeks following a severe exacerbation is required if these biomarkers are used to guide the ongoing management of patients with asthma.
\end{abstract}

Trial registration number Post-results; The Australia New Zealand Trial Registry, >ACTRN12614000443695.

\section{INTRODUCTION}

Peripheral blood eosinophils, fractional exhaled nitric oxide (FeNO), serum periostin and serum IgE are all markers of type-2 inflammation in asthma. Peripheral blood eosinophils, FeNO and serum periostin are predictors of response to treatment with monoclonal antibody therapy directed against IgE, ${ }^{1}$ Interleukin (IL) 4 receptor-alpha, ${ }^{2}$ IL- $5^{3}$ and IL-13. ${ }^{45}$ Blood eosinophil levels are used to determine eligibility for monoclonal antibody therapy directed against IL- $5,{ }^{6}$ and monoclonal antibody therapy directed against IgE is indicated in patients within a target serum IgE range. ${ }^{7}$ The time course of changes of these collective biomarkers during and after a severe exacerbation of asthma have not previously been described. We have established the time course of changes in these biomarkers in the 12 weeks after a severe asthma exacerbation and treatment thereof.

\section{METHODS}

The full methods, results and their interpretation are detailed in the online supplementary file. In summary, we recruited 34 adults aged 18-75 years, from the Wellington Hospital Emergency Department and participating general practitioners. The participants presented within 24 hours of commencing systemic corticosteroid therapy for a severe exacerbation of asthma, as defined by the American Thoracic Society/European Respiratory Society. ${ }^{8}$ Courses of corticosteroids separated by more than a week were treated as separate severe exacerbations. The trial was prospectively registered with the Australia New Zealand Trial Registry (ACTRN12614000443695).
At enrolment, the participants completed the Asthma Control Questionnaire-5, undertook spirometry and assessment of FeNO and blood samples for blood eosinophils, serum IgE and serum periostin. Further visits took place at 1, 2, 4, 8 and 12 weeks, with repeat measurements undertaken as outlined in the study plan (online supplementary table S1). If a participant suffered a further exacerbation after enrolment, this was treated as a new exacerbation for the purposes of analysis. The severe exacerbation onset was marked as the day of commencement of systemic corticosteroids.

Normality assumptions for FeNO, serum periostin and serum IgE were not met, so these variables were analysed on a logarithm-transformed scale. Mixed linear models were used to compare the reference 12-week biomarker measurement with each repeated measure, using a repeated measures design. A type I error rate of 0.5 and associated 95\% CI are shown, for individual statistical tests, SAS V.9.4 was used.

\section{RESULTS}

The flow of participants is shown in online supplementary figure S2. A total of 34 participants enrolled in the study as described in online supplementary table S2. The average length of systemic corticosteroid prescription was 10.9 days (range 1-28 days) with the participants taking an average dose of $36 \mathrm{mg}$ of prednisone per day (range 20-60 mg per day). Most participants had a single severe exacerbation over the 12 weeks $(25 / 34,74 \%)$, with six, two and one participant having two, three and four severe exacerbations, respectively, resulting in 43 exacerbations in total.

The time course of the changes in blood eosinophils, FeNO, serum periostin and serum IgE all varied during and after treatment for a severe exacerbation of asthma (table 1, figure 1A-D). Blood eosinophils, serum periostin and FeNO fell 1 day, 1 week and 2 weeks after treatment with systemic corticosteroids, respectively. In contrast the initial serum IgE measurement was higher than the reference week 12 value. All the biomarkers achieved a relatively stable level within $4-8$ weeks after the start of a severe exacerbation.

\section{DISCUSSION}

These findings demonstrate that the magnitude and duration of change in type- 2 biomarkers 


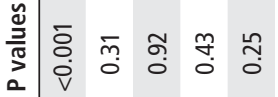

ธิ

茴

তิ一

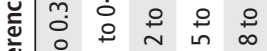

竞

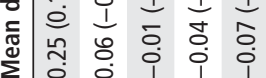

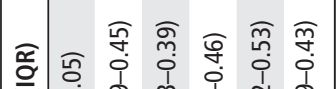
च 营递过 帘

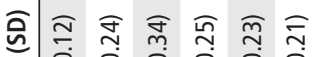
ᄃำ 焉

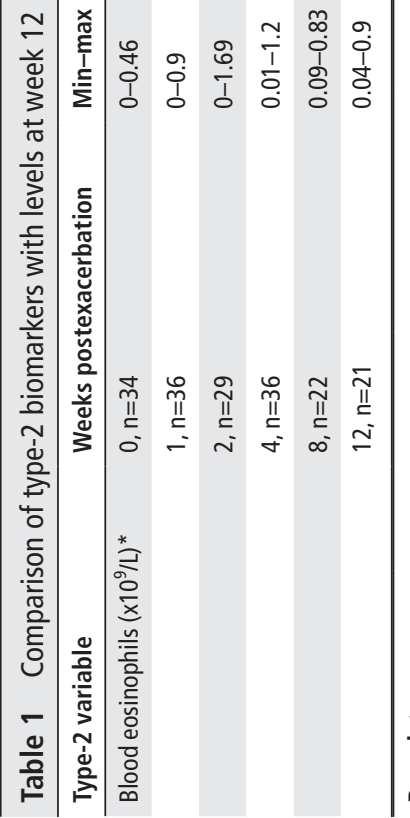

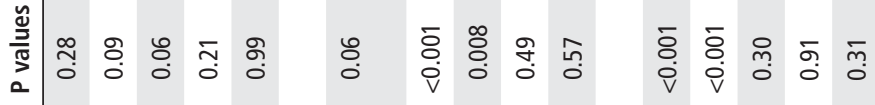

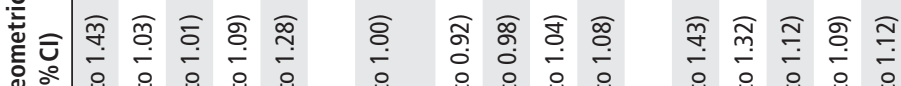
英 㗄

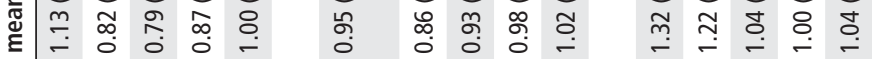
ษิ

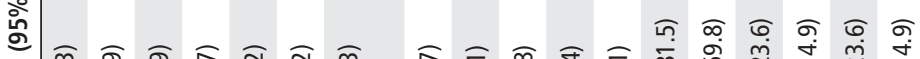

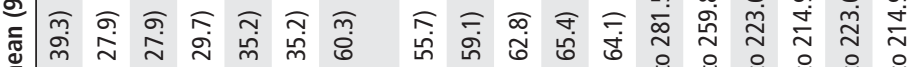
E

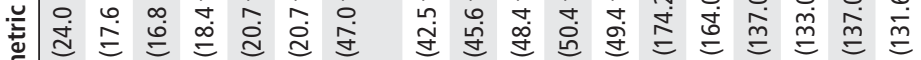

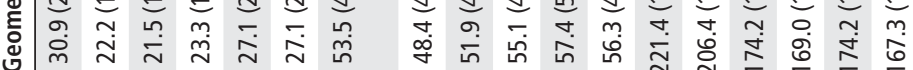

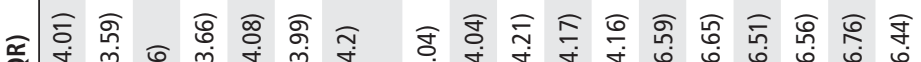
年

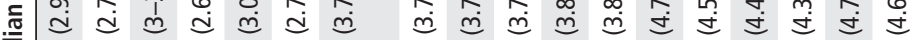

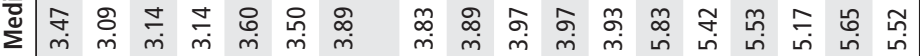

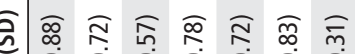

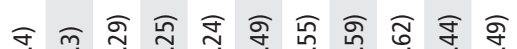

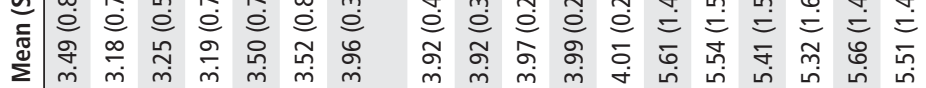

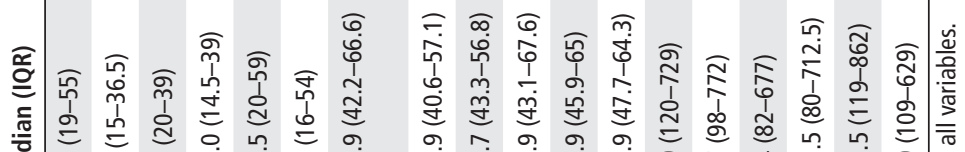

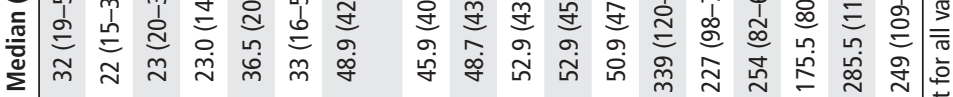

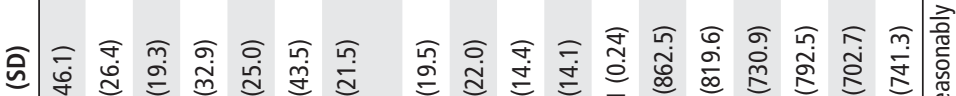
$\stackrel{0}{\frac{0}{x}}$ 竞

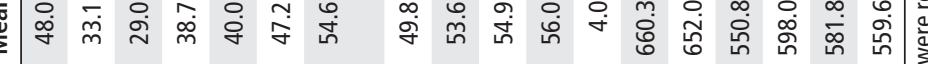

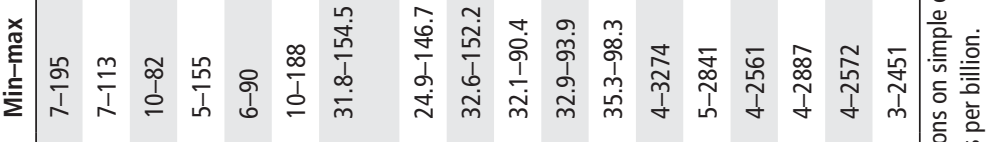

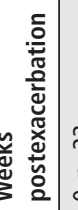

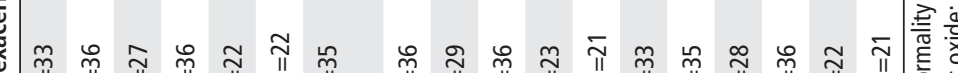
竞

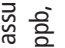
吾离

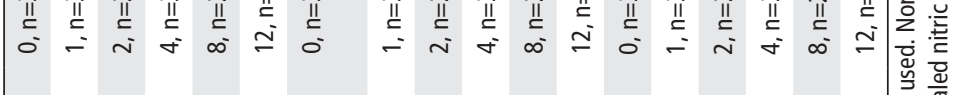

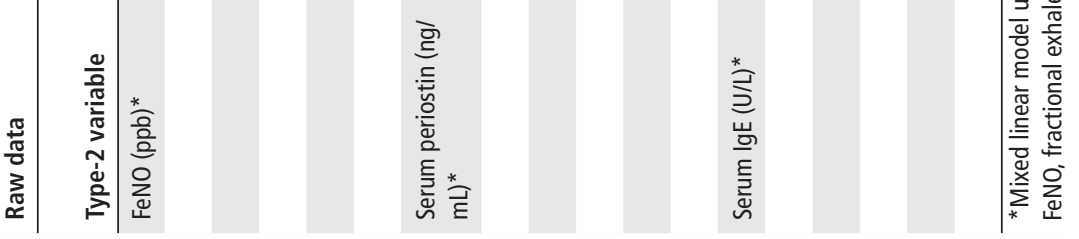



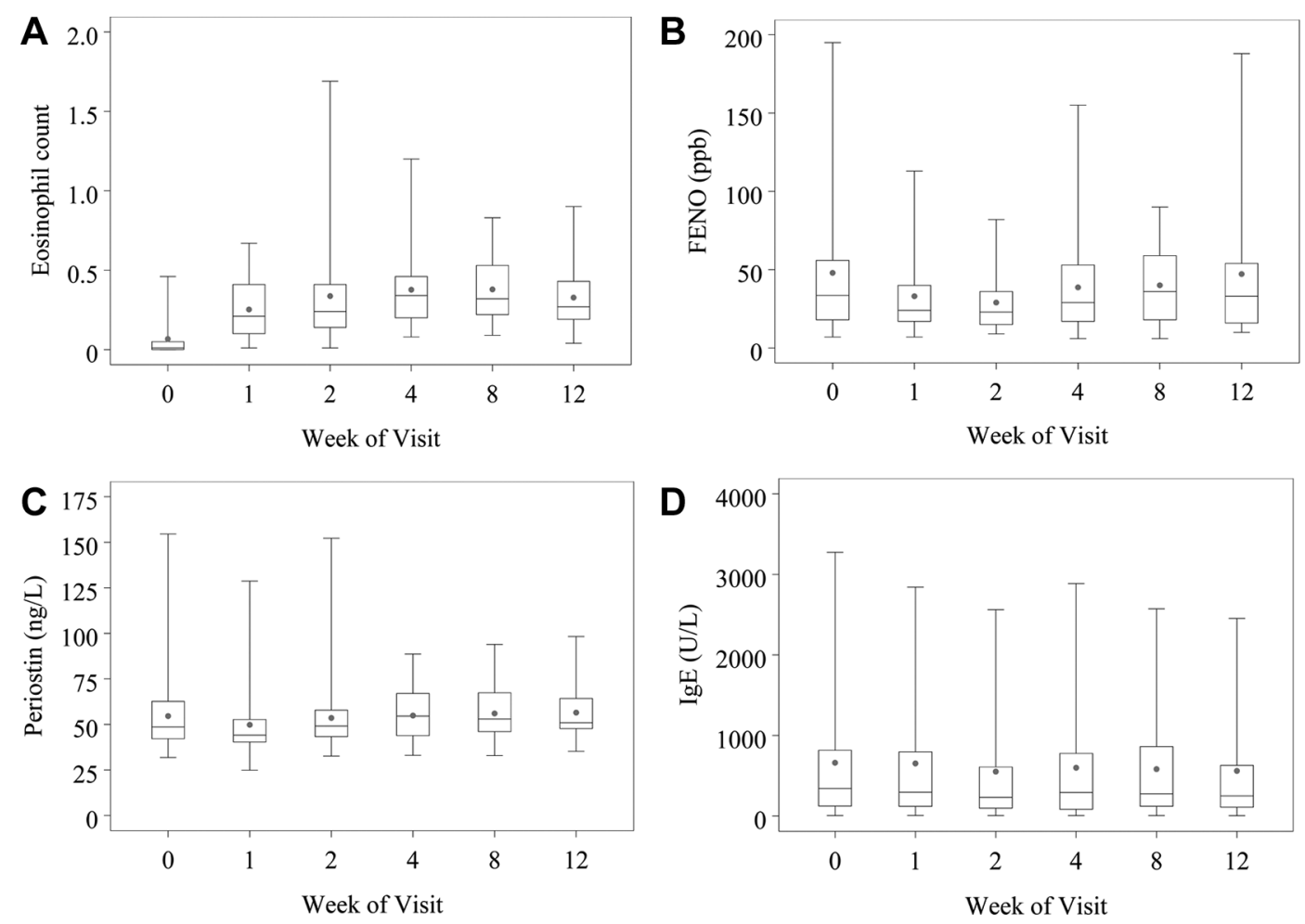

Figure 1 Boxplot of (A) peripheral blood eosinophils, (B) fractional exhaled nitric oxide (FeNO), (C) serum periostin and (D) serum IgE by week after an exacerbation.

vary during and after a severe exacerbation, and recent exacerbation history should be considered in order to understand the magnitude of such changes, if these biomarkers are to be used in clinical practice for patient management or treatment decisions. These differences are likely due to different pathways for synthesis and migration between vascular and tissue compartments, lifespans and responses to steroid treatment of each biomarker.

Our study has some methodological limitations. First, a clinically important change in the different type-2 biomarkers has not been determined, although this does not affect the main conclusions of the study. This study was designed to detect a difference in serum periostin, and it is likely that the sample size was too small to detect a meaningful change with respect to FeNO which has greater intraparticipant variability compared with periostin. ${ }^{4}$ Due to the large number of statistical tests, some of the statistically significant associations may be a result of type 1 error rate inflation. Baseline measurements of the type- 2 biomarkers and lung function during a stable disease phase prior to the exacerbation were not obtained, as enrolment was dependent on the participant having started systemic corticosteroids for a severe exacerbation. We, therefore, compared all periostin levels with those taken 12 weeks after the exacerbation under the assumption that levels would have returned to normal by then. The 12 weeks may not be a 'true baseline' due to factors such as improved adherence to inhaled corticosteroid (ICS) treatment or increase in ICS dose which are known to occur after an acute exacerbation and could potentially affect biomarker levels. Our study could be subject to selection bias, as the participants who had a single exacerbation $(n=25)$ and were followed up for the duration of the 12-week study may have a different biomarker profile to those who had multiple exacerbations $(n=9)$ or who were lost to follow-up $(n=5)$. Finally, the underlying cause of the asthma exacerbations were heterogeneous. Different triggers (viral, allergen or bacterial) can initiate different inflammatory cascades, ${ }^{9}$ but this study was not powered to analyse the difference in response between these sub-groups.

In conclusion, peripheral blood eosinophils, FeNO, serum periostin and serum IgE have different time courses and magnitude of change during and following treatment for a severe exacerbation of asthma. These findings provide further evidence in support of their heterogeneity in asthma and suggest that a delay of up to 8 weeks following a severe exacerbation is required if these collective biomarkers are used in clinical practice to guide patient management and treatment decisions.

Acknowledgements We thank the study participants for their involvement in the study.

Contributors $C T J H, J F, M W, R B$ and IB conceived the idea for and designed the study. RS, NS, SB, AS and RV collected the data. MW did the analysis. RS, CTJH, JF, $M W, R B$ and IB were involved in data interpretation. RS, NS, SE, AS, RV, CTJH, JF, MW, $\mathrm{RB}$ and IB drafted the report. RS, NS, SE, AS, RV, CTJH, JF, MW, RB and IB critically reviewed and revised the report.

Funding This study was funded by Genentech Inc, Members of Genentech were involved in study design, data interpretation and the drafting of this manuscript, but had no influence in the decision to publish the manuscript or disseminate the findings

Competing interests $\mathrm{CH}$ and JM are employees of Genentech Inc., a member of the Roche group. RB reports grants from Genentech during the conduct of the study; personal fees from Health Research Council of New Zealand, Glaxo Smith Kline, AstraZeneca, and Novartis outside the submitted work; grants from AstraZeneca, Chiese, Cephalon, Genentech, Novartis and Sanofi Aventis outside the submitted work. Other authors have no competing interests to declare.

Patient consent Not required.

Ethics approval Ethical approval was given by the New Zealand Health and Disability Ethics Committee (13/NTB/191). 
Provenance and peer review Not commissioned; externally peer reviewed.

Data sharing statement De-identified data are available from the corresponding author on reasonable request.

\section{REFERENCES}

1 Hanania NA, Wenzel S, Rosén $\mathrm{K}$, et al. Exploring the effects of omalizumab in allergic asthma: an analysis of biomarkers in the EXTRA study. Am J Respir Crit Care Med 2013;187:804-11.

2 Wenzel S, Swanson B, Teper A, et al. Dupilumab reduces severe exacerbations in periostin-high and periostin-low asthma patients. Eur Respir J 2016;48:0A1798.

3 Ortega HG, Yancey SW, Mayer B, et al. Severe eosinophilic asthma treated with mepolizumab stratified by baseline eosinophil thresholds: a secondary analysis of the DREAM and MENSA studies. Lancet Respir Med 2016;4:549-56.
4 Corren J, Lemanske RF, Hanania NA, et al. Lebrikizumab Treatment in Adults with Asthma. N Engl J Med Overseas Ed 2011;365:1088-98.

5 Brightling $C E$, Chanez $P$, Leigh $R$, et al. Efficacy and safety of tralokinumab in patients with severe uncontrolled asthma: a randomised, double-blind, placebo-controlled, phase 2b trial. Lancet Respir Med 2015;3:692-701.

6 Ortega HG, Liu MC, Pavord ID, et al. Mepolizumab Treatment in Patients with Severe Eosinophilic Asthma. N Engl J Med Overseas Ed 2014;371:1198-207.

7 Bousquet J, Rabe K, Humbert M, et al. Predicting and evaluating response to omalizumab in patients with severe allergic asthma. Respir Med 2007;101:1483-92.

8 Reddel HK, Taylor DR, Bateman ED, et al. An official American Thoracic Society/ European Respiratory Society statement: asthma control and exacerbations: standardizing endpoints for clinical asthma trials and clinical practice. Am J Respir Crit Care Med 2009:180:59-99.

9 Robinson D, Humbert M, Buhl R, et al. Revisiting Type 2-high and Type 2-low airway inflammation in asthma: current knowledge and therapeutic implications. Clinical \& Experimental Allergy 2017:47:161-75. 